\title{
Voxel based morphometry of grey matter abnormalities in patients with medically intractable temporal lobe epilepsy: effects of side of seizure onset and epilepsy duration
}

\author{
S S Keller, U C Wieshmann, C E Mackay, C E Denby, J Webb, N Roberts
}

J Neurol Neurosurg Psychiatry 2002;73:648-656

See end of article for authors' affiliations .....................

Correspondence to: Dr S Keller, MARIARC, University of Liverpool, Pembroke Place, Liverpool L69 3BX, UK:

kellers@liv.ac.uk

Received 19 April 2002 In revised form 12 August 2002

Accepted 20 August 2002
Objectives: To investigate the use of whole brain voxel based morphometry (VBM) and stereological analysis to study brain morphology in patients with medically intractable temporal lobe epilepsy; and to determine the relation between side, duration, and age of onset of temporal lobe epilepsy, history of childhood febrile convulsions, and grey matter structure.

Methods: Three dimensional magnetic resonance images were obtained from 58 patients with left sided seizure onset (LSSO) and 58 patients with right sided seizure onset (RSSO), defined using EEG and foramen ovale recordings in the course of presurgical evaluation for temporal lobectomy. Fifty eight normal controls formed a comparison group. VBM was used to characterise whole brain grey matter concentration, while the Cavalieri method of modern design stereology in conjunction with point counting was used to estimate hippocampal and amygdala volume. Age and sex were used as confounding covariates in analyses.

Results: LSSO and RSSO patients showed significant reductions in volume (using stereology) and grey matter concentration (using VBM) of the hippocampus, but not of the amygdala, in the presumed epileptogenic zone when compared with controls, but hippocampal (and amygdala) volume and grey matter concentration were not related to duration or age of onset of epilepsy. LSSO and RSSO patients with a history of childhood febrile convulsions had reduced hippocampal volumes in the presumed epileptogenic zone compared with patients without such a history. Left amygdala volume was also reduced in LSSO patients with a history of childhood convulsions. VBM results indicated bilateral thalamic, prefrontal, and cerebellar GMC reduction in patients, which correlated with duration and age of onset of epilepsy.

Conclusions: Hippocampal sclerosis is not necessarily the consequence of recurrent temporal lobe seizures. A major cause of hippocampal sclerosis appears to be an early aberrant neurological insult, such as childhood febrile seizures. Secondary brain abnormalities exist in regions outside the presumed epileptogenic zone and may result from recurrent seizures.
$\mathrm{H}$ ippocampal sclerosis is the most common finding in patients with medically intractable temporal lobe epilepsy. ${ }^{1}$ However, the relation between the duration of epilepsy and hippocampal sclerosis is not fully understood. Some cross sectional research has found evidence for progressive hippocampal volume loss in patients with temporal lobe epilepsy, ${ }^{2-5}$ suggesting that hippocampal atrophy is a consequence of recurrent temporal lobe seizures. Conversely, others have found no relation between hippocampal volume and duration of epilepsy, ${ }^{67}$ which suggests that hippocampal abnormalities do not result from repeated seizures. Hippocampal sclerosis has been related to the age of onset of epilepsy and a history of childhood febrile convulsions in some studies. $^{37}$ However, although atrophy of the amygdala, ${ }^{89}$ entorhinal cortex, ${ }^{10-12}$ parahippocampal gyrus, ${ }^{13}{ }^{14}$ lateral temporal lobe, ${ }^{15}{ }^{16}$ and thalamus ${ }^{17}$ have been associated with temporal lobe epilepsy, there is inadequate understanding of whether extrahippocampal atrophy is related to these clinical variables.

Previous magnetic resonance (MR) image analysis techniques have employed region of interest measurements to investigate the relation between hippocampal volume and duration of epilepsy, age of onset, and febrile convulsions. In the present study, we have used whole brain voxel based morphometry (VBM), which automatically characterises grey matter concentration on magnetic resonance (MR) images.
VBM allows the investigation of grey matter abnormalities with no a priori region of interest, enabling a comprehensive and even handed analysis of global brain structure. ${ }^{18}$ VBM has previously been used to indicate region specific grey matter abnormalities in patients with temporal lobe epilepsy and hippocampal atrophy, ${ }^{19}$ juvenile myoclonic epilepsy, ${ }^{20}$ affective aggression and temporal lobe epilepsy, ${ }^{21}$ and malformations of cortical development causing epilepsy. ${ }^{22}$ In particular, we performed voxelwise statistical analyses on grey matter concentration of patients with left sided seizure onset (LSSO) and right sided seizure onset (RSSO), defined by EEG and invasive foramen ovale recordings. We also investigated the effects of temporal lobe epilepsy on hippocampal and amygdala volume, which were estimated by application of the Cavalieri method of modern design stereology in conjunction with point counting. The distribution of grey matter loss was investigated with respect to laterality of seizure onset, duration of epilepsy, age of onset, and history of febrile convulsions.

\section{METHODS}

Participants and clinical information

Laterality of seizure onset (presumed epileptogenic zone) was determined from EEG recordings, and from invasive foramen ovale recordings when EEG recordings were non-localising. Among 156 patients under evaluation for surgical remediation 


\begin{tabular}{|c|c|c|c|}
\hline Characteristic & LSSO & RSSO & Controls \\
\hline $\mathrm{n}$ & 58 & 58 & 58 \\
\hline Male & 34 & 29 & 30 \\
\hline Female & 24 & 29 & 28 \\
\hline Age (years) & 34 (19 to 55$)$ & $34(20$ to 51$)$ & 40 (22 to 60$)$ \\
\hline $\begin{array}{l}\text { Age of onset of } \\
\text { epilepsy (years) }\end{array}$ & $10(1$ to 34$)$ & 11 (1 to 34$)$ & - \\
\hline $\begin{array}{l}\text { Duration of epilepsy } \\
\text { (years) }\end{array}$ & 24 (2 to 47$)$ & 24 (4 to 48$)$ & - \\
\hline 1 to 10 years $(n)$ & 11 & 8 & - \\
\hline 11 to 20 years $(n)$ & 13 & 11 & - \\
\hline 21 to 30 years $(n)$ & 13 & 21 & - \\
\hline$>30$ years $(n)$ & 21 & 18 & - \\
\hline $\begin{array}{l}\text { History of febrile } \\
\text { convulsions (n) }\end{array}$ & 22 & 18 & - \\
\hline
\end{tabular}

of complex partial seizures with temporal lobe origin, 58 had LSSO and 58 had RSSO. Patients with bilateral seizure onset $(\mathrm{n}=19)$ and for whom side of seizure onset could not be determined $(n=21)$ were excluded from the analyses. Table 1 gives demographic and clinical information on the patients. Fifty eight subjects with no history of neurological or psychiatric symptoms were used as a control population.

\section{MR image acquisition}

Tl weighted images for all patients and controls were obtained with a 1.5T SIGNA whole body MR imaging system (GE Medical Systems, Milwaukee, Wisconsin, USA). A spoiled gradient echo (SPGR) pulse sequence (time of echo $(\mathrm{TE})=9$ $\mathrm{ms}$, time of repetition $(\mathrm{TR})=34 \mathrm{~ms}$, flip angle $=30^{\circ}$ ) produced 124 coronal $\mathrm{Tl}$ weighted images with a field of view (FOV) of $20 \mathrm{~cm}$. Each image refers to a contiguous section of tissue of $1.6 \mathrm{~mm}$ thickness. Acquisition time was 13 minutes and 56 seconds for a 1 NEX scan.

\section{MR image analysis: stereology}

The Cavalieri method in combination with point counting was used to estimate the volumes of left and right hippocampus and amygdala in all participants. ${ }^{19}{ }^{23} 24$ For analysis, the three dimensional MR images were transferred to ANALYZE (Mayo Foundation, Minneapolis, Minnesota, USA) on a SPARC 10 workstation (Sun Microsystems, Mountain View, California, USA), and the $256 \times 256 \times 124$ acquired voxels of side $0.781 \times$ $0.781 \times 1.6 \mathrm{~mm}$ were linearly interpolated to $256 \times 256 \times 256$ cubic voxels of side $0.781 \mathrm{~mm}$. Image sections were reformatted perpendicular to the long axis of the hippocampus to provide optimal visualisation of medial temporal lobe structures. The direction of reformatting was adjusted so that the brain appeared symmetrical on the reformatted sections.

The hippocampus as defined in the present study comprised hippocampus proper, dentate gyrus, ambient gyrus, subiculum, fimbria, alveus, and hippocampal vertical digitations. We excluded the uncus and the choroid plexus. The hindmost section of the hippocampus was defined as the section on which the lateral ventricles divide into frontal and temporal horns. On the most anterior section, the hippocampus was differentiated from the amygdala by visualisation of the alveus and typically additionally by a region of cerebrospinal fluid (CSF) superior to the alveus. Anteriorly, measurement of the amygdala stopped at the last image on which its boundary with the adjacent white matter of the temporal lobe could clearly be identified

Volume estimation was achieved through sampling a series of equally spaced MR images, beginning with a random starting position. Each image was overlain with a test system comprising a regular array of test points, and the number of points lying within each transect through the hippocampus and amygdala was recorded. We did not employ a random grid orientation as difficulties are encountered in representing tilted test systems as relatively small brain structures within the image pixel matrix. However, orientation of the test system does not affect the unbiased nature of the volume estimate. Separation between test points on the square grid used for point counting was $0.234 \mathrm{~cm}$ (that is, three pixels) and slice interval was also $0.234 \mathrm{~cm}$ for the hippocampus. The same grid spacing was used for the amygdala but with a slice interval of $0.156 \mathrm{~cm}$ (two pixels). Unbiased estimates of transect area were obtained by multiplying the total number of points recorded by the area corresponding to each test point (for example, $0.234 \times 0.234=0.0548 \mathrm{~cm}^{2}$ for the hippocampus). An unbiased estimate of structure volume was obtained as the sum of the estimated areas of the structure transects on consecutive systematic sections multiplied by the distance between sections. For the hippocampus, approximately 150 points were recorded on 10 to 15 systematic random sections, while for the amygdala approximately 120 points were recorded on eight to 10 systematic random sections. Sectioning and point counting intensities were optimised to achieve a coefficient of error on the Cavalieri volume estimates of between $3 \%$ and 5\%, as described by Roberts et al. ${ }^{24}$ An inter/intrarater reliability study was carried out by three raters who estimated hippocampal volumes. Intraclass correlation coefficients were calculated and were greater than 0.9 .

\section{MR image analysis: voxel based morphometry}

Data were analysed in SPM99 (Wellcome Department of Cognitive Neurology, www.fil.ion.ucl.ac.uk) running in MATLAB 5.3 (The MathWorks, Natick, Massachusetts, USA) on a Sun Ultra 10 workstation (Sun Microsystems). The technique of VBM used in the present study is illustrated in fig 1.

\section{Spatial normalisation}

To enable intergroup comparisons, VBM corrects for interindividual variability in brain size by spatially normalising each three dimensional MR image to a reference template (fig 1, steps 1-2). The images of all patients and controls were normalised to the 305 normal dataset of the Montreal Neurological Institute (MNI), which approximates the stereotaxic space defined in the atlas of Talairach and Tournoux. ${ }^{25}$ This included a 12 parameter affine transformation and non-linear normalisation using 1176 parameters to model brain shape. Brains were not masked during normalisation. Normalised images were transformed from voxel size $0.781 \times 0.781 \times 1.6$ $\mathrm{mm}$ to $1 \mathrm{~mm} \times 1 \mathrm{~mm} \times 1 \mathrm{~mm}$.

\section{Segmentation and extraction}

Normalised images were segmented into grey matter, white matter, and CSF partitions based on voxel intensities and a priori knowledge of the distribution of brain tissue compartments using the 305 MNI template (fig 1, step 3). Images underwent automatic correction for intensity nonuniformity caused by radio frequency inhomogeneity of MR scanning, as prescribed in SPM software. ${ }^{18}$ Some VBM studies have reported the misclassification of non-brain voxels as grey matter owing to similar voxel intensities of grey matter and scalp, diploic space, and sagittal sinus. ${ }^{26}{ }^{27}$ In order to overcome the misclassification of non-brain voxels, a fully automated brain extraction step was included (fig 1, step 4), which removed unconnected non-brain voxels from grey matter images (erosion followed by conditional dilatation ${ }^{27}$ ).

\section{Smoothing}

Normalised grey matter images were smoothed with an isotropic Gaussian kernel with full width half maximum of 10 mm (fig 1, step 5). Convolving grey matter images with the smoothing kernel allowed for interindividual variation in 


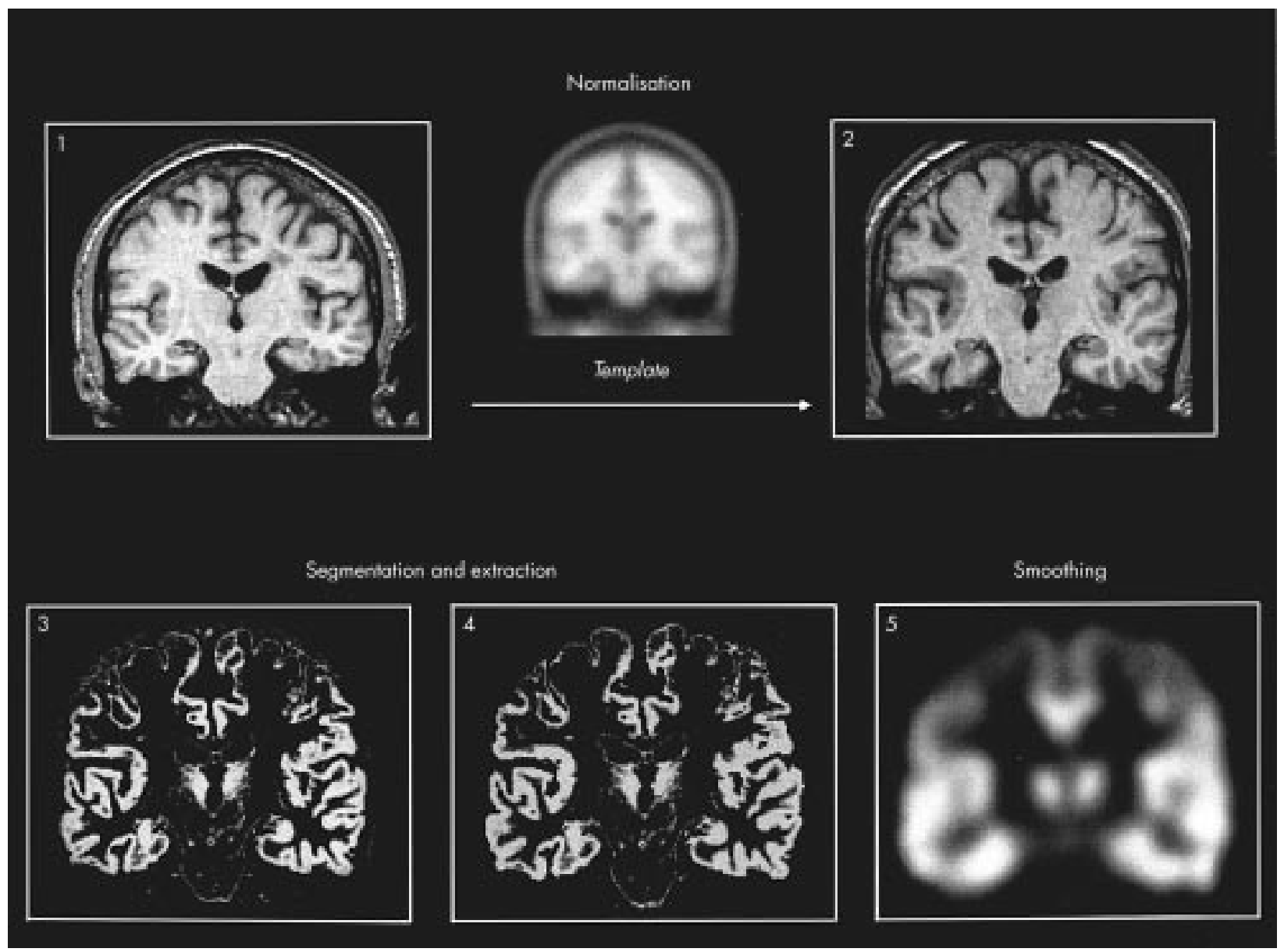

Figure 1 Spatial preprocessing for voxel based morphometry. Raw images (1) are normalised to stereotaxic space (2) using the 305 normal data set of Montreal Neurological Institute (template). Grey matter is automatically segmented from normalised images using a combined voxel intensity and a priori knowledge approach (3). To remove non-grey-matter voxels, an extraction technique is included (4). Normalised grey matter images are smoothed with an isotropic Gaussian kernel with full width half maximum of $10 \mathrm{~mm}(5)$. Bilateral hippocampal pathology and diminished left parahippocampal-fusiform grey/white matter demarcation is apparent in all stages of spatial preprocessing.

gyral anatomy and rendered data more normally distributed for subsequent voxelwise analyses. ${ }^{18}$ The intensity in each voxel of the smoothed image was a local weighted average of grey matter from adjacent voxels. Smoothed grey matter images were considered to represent the local volume of grey matter. ${ }^{20} 22$

\section{Statistical analysis}

The general linear model was used to identify regionally specific differences in grey matter concentration between and within groups. For group comparisons, the images of patients with LSSO and RSSO and controls were compared. Comparisons were also made between patients with and without febrile convulsions. Global effects of duration of epilepsy and age of onset on grey matter concentration were examined in LSSO and RSSO patients in a multiple regression model, including age and sex as confounding covariates.

Contrasts were defined to assess regionally specific differences in grey matter concentration between groups (for group comparisons) and within groups (for regression analyses). Contrasts referred to reductions and increases in grey matter concentration in a subject group, based on the probability of a voxel being grey matter. The output for each comparison is a statistical parametric map of the $t$ statistic $\left(\mathrm{SPM}_{\{t}\right)$, which was transformed to a normal distribution $\left(\operatorname{SPM}_{\{z\}}\right)$. For all analyses, differences in grey matter concentration in brain regions without an a priori hypothesis were only considered significant if they survived a statistical threshold of $p<0.05$ (corrected for multiple comparisons). Brain regions that are hypothesised to show a difference may be reported at uncorrected statistical thresholds. ${ }^{28}$ We considered effects in hippocampal and amygdala regions as significant at an uncorrected height threshold of $\mathrm{p}<0.05$. All results were transformed from MNI space to Talairach space using in-house software (mri3dX, www.mariarc.liv.ac.uk).

\section{RESULTS}

There were no significant differences in duration of epilepsy $(t=0.99 ; \mathrm{p}<0.32)$, age of onset $(t=-0.40 ; \mathrm{p}<0.68)$, or age $(t=0.70 ; \mathrm{p}<0.96)$ between patients with LSSO and RSSO. In all patients, there were significant correlations between the duration of epilepsy and age of onset $(r=-0.39$; p $<0.01)$ and duration of epilepsy and age $(r=0.71 ; \mathrm{p}<0.001)$. Age of onset was related to age, albeit not significantly $(r=-0.21$; $\mathrm{p}<0.07)$.

\section{Stereology}

Mean estimated hippocampal and amygdala volumes in controls and patients are presented in table 2 and fig 2. In controls, the estimated volume of the right hippocampus was greater than that of the left hippocampus $(t=1.97 ; \mathrm{p}<0.05)$. The left hippocampus was smaller in LSSO patients than in the controls $(t=4.37 ; \mathrm{p}<0.001)$, while the right hippocampus was smaller in RSSO patients than in the controls $(t=4.24 ; \mathrm{p}<0.001)$. There were no significant differences in estimated volume of the hippocampus contralateral to the 
Table 2 Estimated hippocampal and amygdala volumes $(\mathrm{ml})$ in all subjects

\begin{tabular}{|c|c|c|c|c|c|c|}
\hline & \multicolumn{2}{|c|}{ Controls } & \multicolumn{2}{|l|}{ LSSO } & \multicolumn{2}{|l|}{ RSSO } \\
\hline & Mean & Range & Mean & Range & Mean & Range \\
\hline L hippocampus & 2.54 & 1.72 to 3.66 & 1.69 & 0.77 to 2.91 & 2.25 & 1.14 to 3.20 \\
\hline R hippocampus & 2.64 & 1.87 to 3.56 & 2.48 & 1.80 to 3.60 & 1.75 & 1.02 to 3.41 \\
\hline L amygdala & 1.90 & 1.43 to 2.61 & 1.83 & 0.87 to 2.94 & 2.03 & 1.03 to 3.08 \\
\hline $\mathrm{R}$ amygdala & 1.94 & 1.42 to 2.54 & 2.03 & 0.97 to 3.13 & 1.83 & 1.08 to 2.80 \\
\hline
\end{tabular}

Values are mean and range.

L, left; LSSO, left sided seizure onset; R, right; RSSO, right sided seizure onset.

epileptogenic focus in patients with LSSO and RSSO compared with the controls. There were no significant differences in the estimated volumes of left and right amygdala between controls, LSSO patients, and RSSO patients.

A multiple regression model, with age and sex used as confounding covariates, showed no significant correlations between estimated hippocampal and amygdala volume and duration of epilepsy and age of onset. Left $(t=2.46 ; \mathrm{p}<0.01)$ and right $(t=2.09 ; \mathrm{p}=0.04)$ hippocampal volumes were significantly smaller in patients who had had febrile convulsions $(\mathrm{n}=40)$ than in those without febrile convulsions $(\mathrm{n}=76)$. When patients were separated according to side of seizure onset, hippocampal volume ipselateral to the epileptogenic focus was reduced in LSSO patients $(\mathrm{n}=22, t=1.98$; $\mathrm{p}<0.05)$ and RSSO patients $(\mathrm{n}=18, t=1.92 ; \mathrm{p}<0.05)$ with previous febrile convulsions compared with patients without febrile convulsions ( $L S S O, \mathrm{n}=36$; RSSO, $\mathrm{n}=40$ ). There was no difference in the volume of the hippocampi contralateral to the epileptogenic focus between patients with and without febrile convulsions. Left amygdala volumes were significantly smaller in LSSO patients with febrile convulsions than in LSSO patients without febrile convulsions $(t=1.99$; $\mathrm{p}<0.05)$.

\section{Voxel based morphometry}

\section{Abnormalities in patients with LSSO and RSSO}

VBM results from group comparisons are presented in table 3. VBM results from all analyses are illustrated in fig 3. Compared with controls, patients had reduced grey matter concentration in bilateral dorsal frontal cortex, left parietooccipital cortex, right thalamus, and cerebellum bilaterally. No reduction in hippocampal grey matter concentration was observed. An increase in grey matter concentration was apparent in the medial orbitofrontal cortex and the temporal lobe bilaterally, in the left cingulate gyrus, and in the right cerebellum.

When analysed separately, both patient groups showed a reduction in grey matter concentration in the hippocampus in the presumed epileptogenic zone. There was no additional reduction in the temporal lobe in either patient group. In both patient groups, reduction in grey matter concentration was apparent in frontal (superior frontal gyrus, middle frontal gyrus), parietal (angular gyrus, supramarginal gyrus, postcentral gyrus), and occipital (superior occipital gyrus, middle occipital gyrus, inferior occipital gyrus) neocortical regions, with LSSO patients showing more widespread reductions. Bilateral reduction in the thalamus was also common to both patient groups. Reduction in the left cerebellum was observed in patients with LSSO, while RSSO patients showed bilateral cerebellar reduction.

A regionally specific increase in grey matter concentration was apparent bilaterally in the medial orbitofrontal cortex in patients with LSSO and RSSO. An increase was observed in the right amygdala, the anterior transverse temporal gyrus, the parahippocampal gyrus, the hippocampus, and the left parahippocampal gyrus in LSSO patients, and in the right
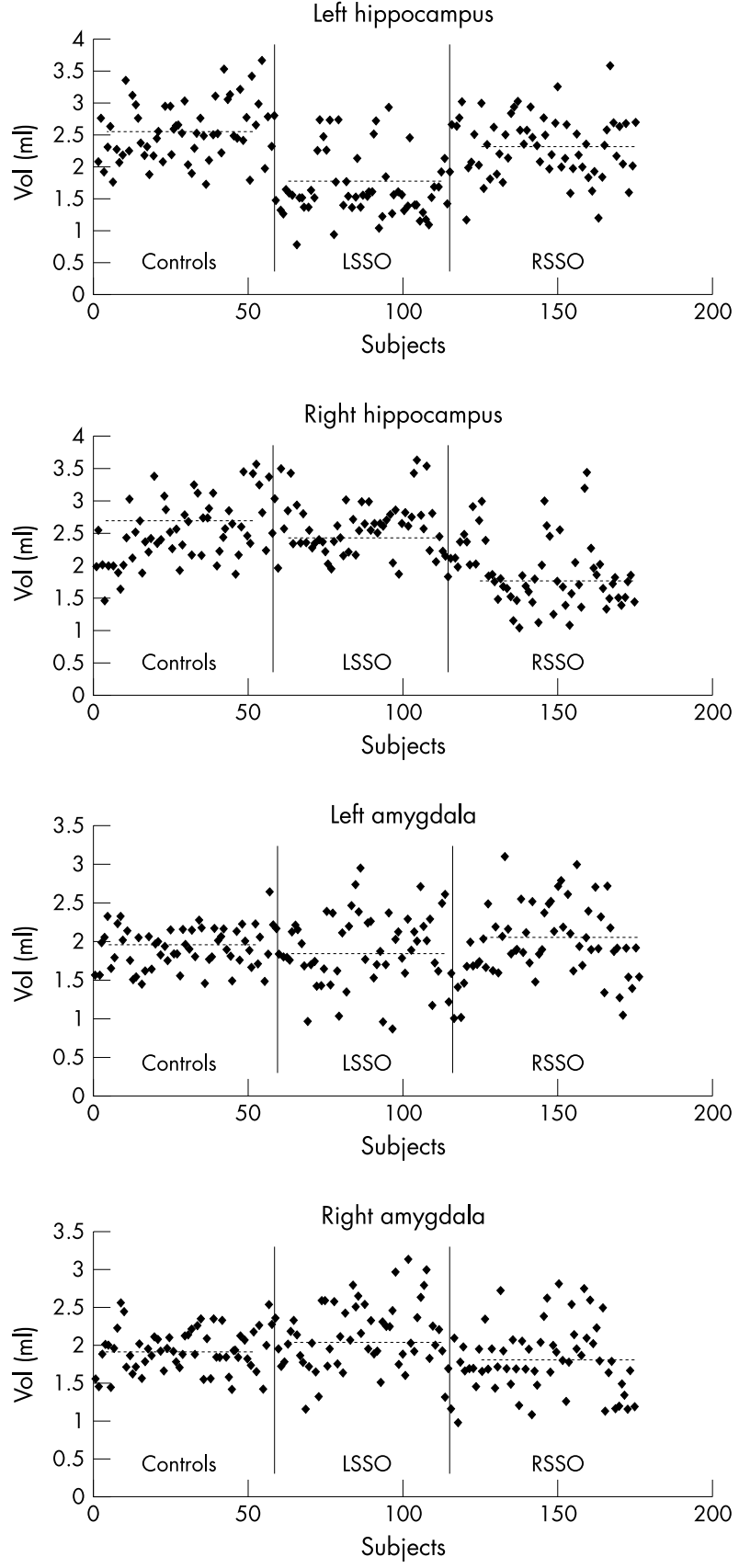

Figure 2 Estimated volumes of left and right hippocampus and amygdala in controls and patients with left sided seizure onset (LSSO) and right sided seizure onset (RSSO).

superior temporal sulcus, the middle temporal gyrus, the parahippocampal gyrus, and the left amygdala in RSSO 
Table 3 Results from voxel based morphometry: group comparison analyses

\begin{tabular}{|c|c|c|c|c|c|c|c|}
\hline \multirow{2}{*}{$\begin{array}{l}\text { Analysis } \\
\text { All patients - controls }\end{array}$} & \multirow{2}{*}{$\begin{array}{l}\text { Contrast } \\
\text { GMC reduction }\end{array}$} & \multirow{2}{*}{$\begin{array}{l}\text { Region } \\
\text { Bilateral SFG MFG }\end{array}$} & \multirow{2}{*}{$\begin{array}{l}\begin{array}{l}\text { Voxel } \\
\text { cluster }\end{array} \\
10012 \\
\end{array}$} & \multirow{2}{*}{$\begin{array}{l}\operatorname{SPM}_{\{z\}} \\
\operatorname{lnf}\end{array}$} & \multicolumn{2}{|c|}{ Peak coordinates } & \multirow[b]{2}{*}{40.2} \\
\hline & & & & & 7.0 & 42.9 & \\
\hline & & Right thalamus & 2778 & 7.55 & 9.9 & -8.4 & 7.8 \\
\hline & & Left AG, SMG, SOG & 3623 & 7.13 & -50.7 & -63.8 & 31.6 \\
\hline & & Right cerebellum & 5071 & 7.02 & 22.9 & -81.6 & -32.1 \\
\hline & & Left cerebellum & 2228 & 6.89 & -37.8 & -71.5 & -25.9 \\
\hline & GMC increase & Bilateral anterior CG, GR, OS, SCG & 6041 & $\operatorname{lnf}$ & -8.0 & 19.9 & -10.2 \\
\hline & & Right STG, IC & 1140 & $\operatorname{lnf}$ & 40.7 & -17.7 & -3.3 \\
\hline & & Left CG & 1214 & 7.44 & -9.9 & 26.4 & 21.7 \\
\hline & & Right PG, EC & 2562 & 7.23 & 29.8 & -22.5 & -19.9 \\
\hline & & Left PG & 2377 & 7.05 & -25.8 & -34.8 & -13.4 \\
\hline & & Right amygdala & 2445 & 6.85 & 18.9 & -7.6 & -15.6 \\
\hline & & Right SFG & 1594 & 6.12 & 23.9 & 59.6 & 2.6 \\
\hline & & Right cerebellum & 1305 & 5.99 & 12.9 & -65.3 & -18.6 \\
\hline & & Left IC & 1568 & 5.87 & -40.8 & 1.5 & 11.0 \\
\hline \multirow{22}{*}{$\begin{array}{l}\text { LSSO patients - } \\
\text { controls }\end{array}$} & GMC reduction & Left hippocampus & 1343 & 7.12 & -28.8 & -14.5 & -16.1 \\
\hline & & Bilateral SFG, MFG, CS & 14286 & 7.01 & 12.9 & 57.1 & 31.2 \\
\hline & & Left MOG, AG & 2818 & 6.48 & -50.7 & -66.5 & 35.4 \\
\hline & & Left MFG & 637 & 6.09 & -45.7 & 27.7 & 29.0 \\
\hline & & Left AG & 311 & 6.04 & -26.8 & -58.7 & 54.3 \\
\hline & & Right AG & 413 & 5.99 & 42.7 & -60.0 & 48.9 \\
\hline & & Right SFG & 516 & 5.92 & 5.0 & -12.9 & 54.8 \\
\hline & & Right thalamus & 716 & 5.74 & 15.9 & -16.2 & 7.2 \\
\hline & & Right IPS & 1052 & 5.69 & 28.8 & -48.6 & 62.1 \\
\hline & & Right IOS & 337 & 5.61 & 35.8 & -76.7 & 26.7 \\
\hline & & Left MFG & 340 & 5.55 & -40.8 & 42.2 & 26.4 \\
\hline & & Left thalamus & 268 & 5.54 & -12.9 & -12.3 & 8.9 \\
\hline & & Left AG, SMG & 939 & 5.44 & -39.8 & -36.7 & 45.9 \\
\hline & & Left cerebellum & 269 & 5.41 & -40.8 & -70.5 & -24.3 \\
\hline & GMC increase & Bilateral anterior CG, GR, OS & 3409 & 6.77 & -8.0 & 19.9 & 0.2 \\
\hline & & Left PG & 1952 & 6.45 & -25.8 & -33.8 & -12.8 \\
\hline & & Right amygdala, GP & 3785 & 6.06 & 15.9 & 0.1 & -15.9 \\
\hline & & Right IC, ATTG & 1114 & 5.77 & 38.8 & -18.7 & -3.3 \\
\hline & & Right cerebellum & 3911 & 5.75 & 17.9 & -58.4 & -17.3 \\
\hline & & Left cerebellum & 3090 & 5.73 & -12.9 & 68.6 & -25.2 \\
\hline & & Left IC & 2045 & 5.59 & -40.8 & 5.2 & 6.2 \\
\hline & & Right PG, hippocampus & 1189 & 5.44 & 29.8 & -24.3 & -18.1 \\
\hline \multirow{21}{*}{$\begin{array}{l}\text { RSSO patients - } \\
\text { controls }\end{array}$} & GMC reduction & Right hippocampus & 1492 & 7.33 & 34.8 & -22.0 & -9.8 \\
\hline & & Right thalamus & 1261 & 6.89 & 15.9 & -16.2 & 7.2 \\
\hline & & Bilateral SFG & 2265 & 6.78 & 20.9 & 30.7 & 49.1 \\
\hline & & Right cerebellum & 9066 & 6.76 & 18.9 & -80.0 & -36.4 \\
\hline & & Right PoCG & 759 & 6.51 & 39.8 & -26.7 & 51.8 \\
\hline & & Right SFG & 405 & 6.23 & 5.0 & -12.8 & 56.7 \\
\hline & & Precuneus, POF & 3244 & 6.02 & 7.0 & -69.4 & 36.4 \\
\hline & & Left IOG & 372 & 5.87 & -14.9 & -97.5 & 1.4 \\
\hline & & Left SOG & 846 & 5.71 & -29.8 & -75.5 & 31.2 \\
\hline & & Left cerebellum & 3181 & 5.59 & -31.8 & -77.3 & -23.9 \\
\hline & & Left thalamus & 363 & 5.51 & -13.9 & -16.2 & 7.2 \\
\hline & & Right MFG & 432 & 5.49 & 36.8 & 8.5 & 52.9 \\
\hline & & Left cerebellum & 463 & 5.47 & -38.8 & -44.2 & -25.5 \\
\hline & GMC increase & Bilateral anterior CG, GR, OS, SCG & 5836 & 6.59 & 4.0 & 34.6 & -10.1 \\
\hline & & Right IC, STS, MTG & 4301 & 6.52 & 43.7 & -15.9 & -6.8 \\
\hline & & Right PG & 2125 & 6.38 & 29.8 & -21.6 & -21.6 \\
\hline & & Left CG & 1177 & 6.27 & -9.9 & 26.4 & 21.7 \\
\hline & & Left amygdala, GP & 1603 & 6.11 & -24.8 & -6.4 & -10.6 \\
\hline & & Bilateral posterior $\mathrm{CG}$ & 1642 & 5.99 & 4.0 & -33.8 & 26.4 \\
\hline & & Right CIS & 1274 & 5.73 & 31.8 & 17.9 & -12.6 \\
\hline & & Right SFG & 1701 & 5.66 & 19.9 & 55.8 & 5.6 \\
\hline
\end{tabular}

Region, spatial extent (voxel cluster), significance extent $\left(\mathrm{SPM}_{[z]}\right)$, and Talairach coordinates of peak effect are indicated. All results are reported at $\mathrm{p}<$ 0.05 (corrected for multiple comparisons).

AG, angular gyrus; ATTG, anterior transverse temporal gyrus; $C G$, cingulate gyrus; CS, cingulate sulcus; CIS, circular insular sulcus; EC, entorhinal cortex; GMC, grey matter concentration; GP, globus pallidus; GR, gyrus rectus; IC, insular cortex; inf, infinite; IOG, inferior occipital gyrus; IOS, intraoccipital sulcus; IPS, intraparietal sulcus; LSSO, left sided seizure onset; MFG, middle frontal gyrus; MOG, middle occipital gyrus; MTG, middle temporal gyrus; OS, olfactory sulcus; PG, parahippocampal gyrus; PoCG, postcentral gyrus; POF, parieto-occipital fissure; RSSO, right sided seizure onset; SCG, subcallosal gyrus; SFG, superior frontal gyrus; SMG, supramarginal gyrus; SOG, superior occipital gyrus; STG, superior temporal gyrus; STS, superior temporal sulcus.

patients. Bilateral increases in grey matter concentration in the cerebellum were observed in LSSO patients and an increase in the posterior regions of the cingulate gyrus in RSSO patients.

\section{History of childhood febrile convulsions}

No significant effects were observed in the extramedial temporal lobe ( $p<0.05$, corrected for multiple comparisons) or the hippocampus and amygdala $(\mathrm{p}<0.05$, uncorrected $)$.
Duration and age of onset of epilepsy

VBM results from regression analyses are presented in table 4 and fig 3. When LSSO and RSSO patients were combined, there were significant correlations between the duration of epilepsy (negative) and the age of onset of epilepsy (positive) and the grey matter concentration in the left and right thalami, bilateral frontoparietal cortex (superior frontal gyrus, middle frontal gyrus, cingulate gyrus, precentral gyrus, postcentral gyrus, and angular gyrus), and cerebellum. When 

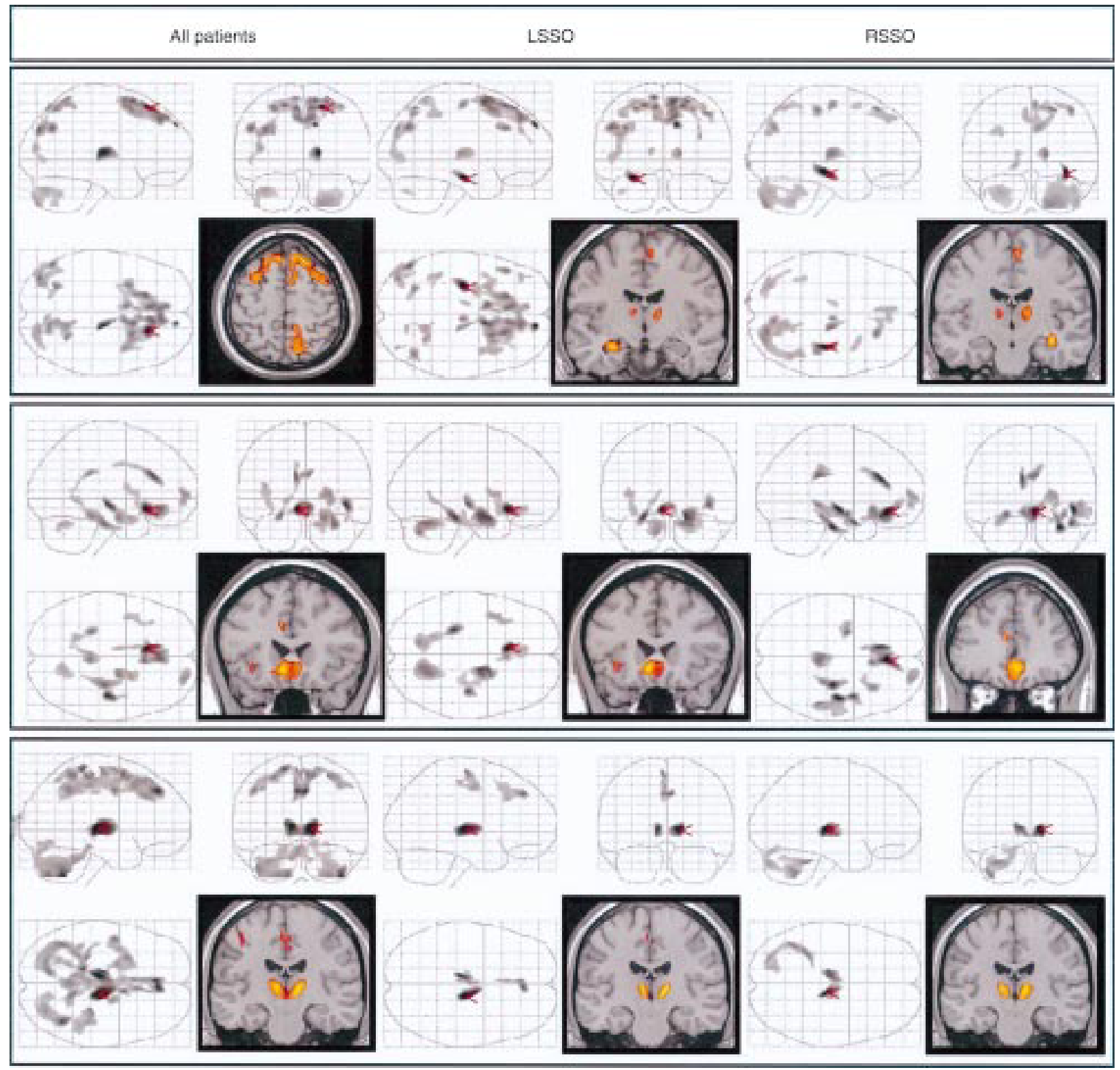

Figure 3 Results from voxel based morphometry. In all analyses, the region of peak effect is indicated using a T1 weighted neuroanatomical template and a red arrow on glass brain projections. Top panel: Grey matter concentration (GMC) reduction in patients relative to controls. Middle panel: GMC increase in patients relative to controls. Bottom panel: Voxelwise correlations between GMC and duration of epilepsy (negative) and age of onset of epilepsy (positive). Results are shown for all patients combined and for left sided seizure onset (LSSO) and right sided seizure onset (RSSO) separately.

analysed separately, correlations were observed between the duration of epilepsy (negative) and the age of onset (positive) and the grey matter concentration in the thalamus bilaterally in LSSO and RSSO patients. Furthermore, grey matter concentration in the medial frontal lobe (superior frontal gyrus and precentral gyrus) in LSSO patients, and in the left cerebellum in RSSO patients, were correlated with duration of epilepsy and age of onset. There were no medial temporal lobe effects at uncorrected statistical thresholds.

\section{DISCUSSION}

Automated whole brain VBM and manual stereology both revealed hippocampal sclerosis ipselateral to the epileptogenic focus in patients with unilateral temporal lobe epilepsy. Furthermore, both techniques suggest that hippocampal sclerosis is not necessarily the consequence of recurrent temporal lobe seizures. Results from stereological analysis of hippocampus and amygdala indicate that a history of febrile convulsions is a major determinant of hippocampal (and amygdala) sclerosis ipselateral to the epileptogenic focus. Furthermore, VBM results indicate secondary grey matter abnormalities of the thalamus, prefrontal cortex, and cerebellum, which correlated with the duration of epilepsy and the age of onset. The biological interpretations and implications of these data are discussed below. First, we will highlight pertinent methodological considerations.

\section{Methodological issues}

There is a large body of research reporting relations between brain structure and duration of epilepsy, age of onset, and history of febrile convulsions, using cross sectional analysis. ${ }^{2-7}$ Some studies have employed longitudinal serial imaging. ${ }^{29} 30$ As suggested by Liu et al, ${ }^{30}$ inferences that can be drawn on disease progression from cross sectional studies are limited, as small changes in anatomical structure over time are often masked by large biological variability across subjects, and 
Table 4 Results from voxel based morphometry: regression analyses

\begin{tabular}{|c|c|c|c|c|c|c|c|}
\hline \multirow{2}{*}{$\begin{array}{l}\text { Analysis } \\
\text { All patients - } \\
D E, A E\end{array}$} & \multirow{2}{*}{$\begin{array}{l}\text { Contrast } \\
\text { GMC } \\
\text { reduction }\end{array}$} & \multirow{2}{*}{$\begin{array}{l}\text { Region } \\
\text { Bilateral thalamus }\end{array}$} & \multirow{2}{*}{$\begin{array}{l}\begin{array}{l}\text { Voxel } \\
\text { cluster }\end{array} \\
6790\end{array}$} & \multirow{2}{*}{$\frac{S P M_{\{z\}}}{7.44}$} & \multicolumn{3}{|c|}{ Peak coordinates } \\
\hline & & & & & 9.9 & -16.4 & 4.5 \\
\hline & & $\begin{array}{l}\text { Bilateral SFG, CG, CS, } \\
\text { PrCG }\end{array}$ & 9234 & 6.56 & 3.0 & 41.8 & 37.5 \\
\hline & & Right cerebellum & 7521 & 6.51 & 40.7 & -56.5 & -37.7 \\
\hline & & Left cerebellum & 7778 & 6.41 & -41.7 & -56.2 & -31.7 \\
\hline & & Left MFG, SFG & 2382 & 6.12 & -46.7 & -1.9 & 41.4 \\
\hline & & Right PoCG, AG & 1786 & 5.67 & 40.7 & -38.5 & 48.7 \\
\hline \multirow{5}{*}{$\begin{array}{l}\text { LSSO patients } \\
-D E, A E\end{array}$} & GMC & & & & & & \\
\hline & reduction & Right thalamus & 2651 & 6.01 & 12.5 & -12.1 & 8.5 \\
\hline & & Left thalamus & 1482 & 5.78 & -12.9 & -12.3 & 8.9 \\
\hline & & Bilateral SFG & 1241 & 5.49 & & 46.2 & 38.6 \\
\hline & & Bilateral PrCG & 948 & 5.45 & -11.9 & -39.8 & 61.7 \\
\hline \multirow{4}{*}{$\begin{array}{l}\text { RSSO patients } \\
-D E, A E\end{array}$} & GMC & & & & & & \\
\hline & reduction & Right thalamus & 2487 & 5.98 & 9.9 & -16.4 & \\
\hline & & Left thalamus & 1528 & 5.76 & -12.9 & -12.3 & 8.9 \\
\hline & & Left cerebellum & 5123 & 5.62 & -41.7 & -56.2 & -31.7 \\
\hline
\end{tabular}

Region, spatial extent (voxel cluster), significance extent $\left(S P M_{\{z}\right)$, and Talairach coordinates of peak effect are indicated. All results are reported at $\mathrm{p}<0.05$ (corrected for multiple comparisons).

$A E$, age of onset of epilapsy; $A G$, angular gyrus; $C G$, cingulate gyrus; $C S$, cingulate sulcus; $D E$, duration of epilepsy; GMC, grey matter concentration; LSSO, left sided seizure onset; MFG, middle frontal gyrus; PoCG, postcentral gyrus; PrCG, precentral gyrus; RSSO, right sided seizure onset; SFG, superior frontal gyrus.

cross sectional studies are unable to give direct information on the causal relation between seizures and structural brain damage. There were several biological and clinical variables not controlled for in the present study, such as seizure frequency, incidence of secondary generalised seizures and status epilepticus, and antiepileptic drug treatment, which have previously been related to brain atrophy. ${ }^{31}$ Serial imaging of individual patients can determine causal relations between individual clinical and biological variables and brain structure. However, longitudinal research has practical and logistical challenges. A comprehensive longitudinal investigation of the time course required to generate brain degeneration is likely to be compromised by participant attrition and evolving MRI equipment.

We were unable to identify clinical differences between patients with short and long duration of epilepsy, which could confound our findings. There remains a possibility of confounding factors including age related atrophy and possible drug effects. Nevertheless, it seems unlikely that these factors could account for the highly lateralised findings observed in our study.

We have shown methodological consistency between automated whole brain VBM and manual region of interest stereology of the hippocampus. Both techniques indicated significant hippocampal grey matter concentration and volume reductions ipselateral to the epileptogenic focus, a consistent finding in patients with unilateral temporal lobe epilepsy. ${ }^{132} 33$ Furthermore, both techniques indicated no significant relations between hippocampal structure and duration of epilepsy, which is also supported by some volumetric studies. ${ }^{67}$ We observed significant hippocampal volume reduction using stereology in patients who had had febrile convulsions relative to patients without febrile convulsions, which is consistent with other work, ${ }^{3}$ but this finding was not replicated by VBM using corrected or uncorrected statistical thresholds. Although VBM is a powerful tool for identifying morphological differences between two distinct subject populations (for example, controls and patients), the technique may be less sensitive in detecting subtle morphological differences between two groups of patients who share similar morphological characteristics (such as hippocampal abnormalities).

\section{Biological implications}

We found no evidence of a relation between hippocampal concentration or volume and duration of epilepsy, which suggests that hippocampal sclerosis-as detected by cross sectional MRI-is not necessarily a consequence of recurrent temporal lobe seizures. Hippocampal sclerosis often precedes temporal lobe epilepsy, as indicated by the presence of this abnormality at birth or during childhood. Van Paesschen et al have suggested $^{29}$ that the hippocampi may be acutely damaged by an aberrant neurological event (such as childhood infection, ischaemia, trauma, or febrile convulsions ${ }^{34}$ ), with progression toward hippocampal sclerosis in the ensuing months and onset of temporal lobe epilepsy months or years later. We cannot, however, exclude the possibility of progressive hippocampal damage, as microscopic hippocampal changes may occur that cannot be resolved by MRI. In some MRI negative patients (that is, patients with no observable hippocampal sclerosis using MRI), hippocampal neuronal loss has been observed on resected specimens. ${ }^{33}$ It is possible that an initial precipitating injury causes hippocampal abnormalities, with progressive deterioration in hippocampal structure and integrity thereafter. Mathern et al suggested that the pathogenesis of temporal lobe epilepsy is associated with an initial precipitating injury that causes damage to the hippocampus before onset of habitual seizures. ${ }^{35}$ Although most hippocampal damage may occur with the initial precipitating injury, there can be progressive hippocampal structural deterioration thereafter, characterised by neuronal loss and axon and synaptic reorganisation.

We have provided evidence indicating abnormalities in brain regions outside the epileptogenic zone in patients with temporal lobe epilepsy. Results of reduced grey matter concentration presented here are consistent with previous studies indicating atrophy of the thalamus, ${ }^{17}{ }^{36}$ frontoparietal cortex, ${ }^{37}$ and cerebellum. ${ }^{38}$ The thalamus has been implicated in the propagation of epileptogenic activity, as it connects the allocortex to the neocortex, and may serve to regulate the spread of epileptogenic discharges from the pathological medial temporal lobe to distant neocortical regions. ${ }^{39-42}$ The prefrontal cortex represents a pathway for interhemispheric propagation of ictal activity and is highly influenced by the epileptogenic zone in the temporal lobe. ${ }^{43}$ The propagation of abnormal neuronal activity throughout these regions may cause grey matter degeneration, although seizure spread to the contralateral temporal lobe is a common pathway and we found no evidence of reduction in temporal lobe grey matter concentration. This is inconsistent with a previous study indicating a negative correlation between duration of epilepsy and 
temporal lobe volume. ${ }^{44}$ Whether recurrent seizures cause brain damage remains a contentious issue. ${ }^{45}$

The significance for postsurgical outcome of grey matter abnormalities outside the epileptogenic zone remains poorly understood. Although two thirds of patients with temporal lobe epilepsy and hippocampal sclerosis will be rendered seizure-free after temporal resection, ${ }^{1}$ seizures will persist in some. The extrahippocampal abnormalities observed in the present VBM study (both reduction and increase in grey matter concentration) may relate to previously described abnormal distributions of grey and white matter, which were associated with poor surgical outcome in patients with hippocampal sclerosis. ${ }^{46}$

Whereas reduction in grey matter concentration most probably corresponds to grey matter atrophy, the neuropathological correlates of an increase in concentration are less clear. Previous reports of increases in the temporal lobes of patients with temporal lobe epilepsy are consistent with the present study. ${ }^{1922}$ Non-atrophic histopathological abnormalities (such as subpial gliosis, subcortical gliosis, or microdysgenesis) causing diminished temporal lobe grey/white matter demarcation are extremely common in patients with temporal lobe epilepsy. ${ }^{47-51}$ An increase in grey matter concentration in the temporal lobe is therefore likely to reflect diminished grey/white matter demarcation (fig 1). Bilateral increases in temporal lobe grey matter in patients with LSSO and RSSO suggest that the neuropathology underlying increases in grey matter concentration is not confined to the epileptogenic zone.

\section{Conclusions}

Hippocampal sclerosis, the most common neuropathological correlate of temporal lobe epilepsy, is not necessarily the consequence of recurrent temporal lobe seizures. However, secondary brain damage, which may be caused by recurrent seizures, exists in regions outside the presumed epileptogenic zone. Our data suggest that severe temporal lobe epilepsy may share some morphological characteristics with neurodegenerative disorders, although the underlying mechanisms may be different. Early medical and, if necessary, surgical minimisation of recurrent seizures may inhibit subsequent extratemporal degeneration and consequent cognitive decline.

\section{ACKNOWLEDGEMENTS}

SSK was funded by a studentship award from Professions Allied to Medicine, the School of Health Sciences, University of Liverpool. We thank the radiographers and nursing staff at MARIARC for their assistance.

\section{Authors' affiliations}

S S Keller, C Denby, N Roberts, The Magnetic Resonance and Image Analysis Research Centre (MARIARC), Pembroke Place, University of Liverpool, Liverpool, UK

U C Wieshmann, The Walton Centre for Neurology and Neurosurgery, Lower Lane, Liverpool, UK

J Webb, C E Mackay, Department of Psychiatry, Warneford Hospital, University of Oxford, Oxford, UK

Competing interests: none declared

\section{REFERENCES}

1 Duncan J. Imaging and epilepsy. Brain 1997;120:339-77.

2 Salamenperä T, Kälviäinen R, Partanen K, et al. Hippocampal damage caused by seizures in temporal lobe epilepsy. Lancet 1998;351:35

3 Salamenperä T, Kälviäinen R, Partanen K, et al. Hippocampal and amygdaloid damage in partial epilepsy. A cross-sectional MRI study of 241 patients. Epilepsy Res 2001;46:69-82.

4 Tasch E, Cendes F, Li L, et al. Neuroimaging evidence of progressive neuronal loss and dysfunction in temporal lobe epilepsy. Ann Neurol 1999;45:568-76.

5 Theodore W, Bhatia S, Hatta J, et al. Hippocampal atrophy, epilepsy duration, and febrile seizures in patients with partial seizures. Neurology 1999;52:132-6.
6 Cendes F, Andermann F, Gloor P, et al. Atrophy of mesial structures in patients with temporal lobe epilepsy: cause or consequence of repeated seizures? Ann Neurol 1993;34:795-801.

7 Davies K, Hermann B, Dohan F, et al. Relationship of hippocampal sclerosis to duration and age of onset of epilepsy, and childhood febrile seizures in temporal lobectomy patients. Epilepsy Res 1996;24:119-26.

8 Guerreiro C, Cendes F, Li L, et al. Clinical patterns of patients with temporal lobe epilepsy and pure amygdala atrophy. Epilepsia 1999;40:453-61

9 Ho S, Consalvo D, Gilliam F, et al. Amygdala atrophy and seizure outcome after temporal lobe epilepsy surgery. Neurology 1998;51:1502-4.

10 Bernasconi N, Bernasconi A, Andermann F, et al. Entorhinal cortex in temporal lobe epilepsy: a quantitative MRI study. Neurology 1999:52:1870-6

11 Bernasconi N, Bernasconi A, Caramanos Z, et al. Entorhinal cortex atrophy in epilepsy patients exhibiting normal hippocampal volumes. Neurology 2001;56:1335-9.

12 Salamenperä T, Kälviäinen R, Partanen K, et al. MRI volumetry of the hippocampus, amygdala, entorhinal cortex, and perirhinal cortex after status epilepticus. Epilepsy Res 2000;40:155-70.

13 Bernasconi N, Bernasconi A, Caramanos Z, et al. Morphometric MRI analysis of the parahippocampal region in temporal lobe epilepsy. Ann NY Acad Sci 2000;911:495-500.

14 Meiners L, Witkamp T, De Kort G, et al. Relevance of temporal lobe white matter changes in hippocampal sclerosis: magnetic resonance imaging and histology. Invest Radiol 1999;34:38-45.

15 Moran N, Lemieux L, Kitchen N, et al. Extrahippocampal temporal lobe atrophy in temporal lobe epilepsy and mesial temporal sclerosis. Brain 2001; 124:167-75.

16 Nishio S, Morioka T, Hisada K, et al. Temporal lobe epilepsy: a clinicopathological study with special reference to temporal neocortical changes. Neurosurg Rev 2000;23:84-9.

17 DeCarli C, Hatta J, Fazilat S, et al. Extratemporal atrophy in patients with complex partial seizures of left temporal origin. Ann Neurol 1998;43:41-5.

18 Ashburner J, Friston K. Voxel-based morphometry - the methods. Neuroimage 2000;11:805-21.

19 Keller S, Mackay C, Barrick T, et al. Voxel-based morphometric comparison of hippocampal and extrahippocampal abnormalities in patients with left and right hippocampal atrophy. Neuroimage 2002;16:23-31.

20 Woermann F, Free S, Koepp S, et al. Abnormal cerebral structure in juvenile myclonic epilepsy demonstrated with voxel-based analysis of MRI. Brain 1999;1 22:2101-7.

21 Woermann F, Van Elst L, Koepp M, et al. Reduction of frontal neocortical grey matter associated with affective aggression in patients with temporal lobe epilepsy: an objective voxel by voxel analysis of automatically segmented MRI. J Neurol Neurosurg Psychiatry 2000;68: 162-9.

22 Woermann F, Free S, Koepp M, et al. Voxel-by-voxel comparison of automatically segmented cerebral gray matter - a rater-independent comparison of structural MRI in patients with epilepsy. Neuroimage 1999;10:373-84.

23 Mackay C, Webb J, Eldridge P, et al. Quantitative magnetic resonance imaging in consecutive patients evaluated for surgical treatment of temporal lobe epilepsy. Magn Reson Imag 2000;18:1 187-99.

24 Roberts N, Garden A, Cruz-Orive L, et al. Estimation of fetal volume by MRI and stereology. Br J Radiol 1994;67:1067-77.

25 Talairach J, Tournoux P. Co-planar stereotaxic atlas of the human brain New York: Thieme, 1998.

26 Baron J-C, Chételat G, Desgranges B, et al. In vivo mapping of grey matter loss with voxel-based morphometry in mild Alzheimer's disease. Neuroimage 2001;14:298-309.

27 Good C, Johnsgrude I, Ashburner J, et al. A voxel-based morphometric study of ageing in 465 normal adult human brains. Neuroimage $2001 ; 14: 21-36$

28 Saykin A, Johnson S, Flashman L, et al. Functional differentiation of medial temporal and frontal regions involved in processing novel and familiar words: an FMRI study. Brain 1999;122:1963-71.

29 Van Paesschen W, Duncan J, Stevens J, et al. Longitudinal quantitative hippocampal magnetic resonance imaging study of adults with newly diagnosed partial seizures: one year follow up. Epilepsia 1998;39:633-9.

30 Liu $\mathbf{R}$, Lemieux L, Bartlett $P$, et al. A longitudinal quantitative MRI study of community-based patients with chronic epilepsy and newly diagnosed seizures: methodology and preliminary findings. Neuroimage $2001 ; 14: 231-43$

31 Lindvall 0 , Nilsson B. Cerebellar atrophy following phenytoin intoxication. Ann Neurol 1984;16:258-60.

32 Cendes F, Andermann F, Gloor P, et al. The role of MRI volumetric studies in the investigation of patients with intractable epilepsy. In: Shorvon S, Fish D, Andermann F, et al, eds. Magnetic resonance scanning and epilepsy. New York: Plenum, 1994:57-61.

33 Kuzneicky R, Jackson G. Magnetic resonance in epilepsy. New York: Raven Press, 1995

34 Vives K, Al-Rodham N, Spencer D. Use of magnetic resonance imaging in surgical strategies for epilepsy. In: Cascino $G$, Jack C, eds. Neuroimaging in epilepsy. Boston: Butterworth-Heinemann, 1996:235-59.

35 Mathern G, Babb T, Leite J, et al. The pathogenic and progressive features of chronic human hippocampal epilepsy. Epilepsia 1996;26:151-61. 
36 Bertam E, Scott C. The pathological substrate of limbic epilepsy: neuronal loss in the medial dorsal thalamic nucleus as the consistent change. Epilepsia 2000;41:S3-8.

37 Marsh L, Morrell M, Shear $P$, et al. Cortical and hippocampal volume deficits in temporal lobe epilepsy. Epilepsia 1997;38:576-87.

38 Sandok E, O'Brien T, Jack C, et al. Significance of cerebellar atrophy in intractable temporal lobe epilepsy: a quantitative MRI study. Epilepsia 2000;41:1315-20

39 Lothman E, Bertram E, Stringer J. Functional anatomy of hippocampal seizures. Prog Neurobiol 1991;37:1-82.

40 Spencer S. Cortical and intercortical seizure spread. In: Meldrum B, Ferendelli J, eds. Current problems in epilepsy, vol 6. Anatomy of epileptogenesis. London: John Libbey, 1988:139-54.

41 Avoli M, Gloor P. Interaction of cortex and thalamus in spike and wave discharges of feline generalized penicillin epilepsy. Exp Neurol 1982; 76:196-217.

42 Saunders R, Rosene D, Van Hoesen G. Comparison of the efferents of the amygdala and the hippocampus formation in the rhesus monkey. II. Reciprocal and non-reciprocal connections. J Comp Neurol 1988;271:185-207.

43 Lieb J, Dasheiff R, Engel J. Role of the frontal lobes in the propagation of mesial temporal lobe seizures. Epilepsia 1991;32:822-37.
44 Lee J, Andermann F, Dubeau F, et al. Morphometric analysis of the temporal lobe in temporal lobe epilepsy. Epilepsia 1998;39:727-36. Holmes G. Do seizures cause brain damage? Epilepsia $1991 ; 32:$ S14-28

46 Sisodiya S, Moran N, Free S, et al. Correlation of widespread preoperative magnetic resonance imaging changes with unsuccessful surgery for hippocampal sclerosis. Ann Neurol 1997:41:490-6.

47 Choi D, Na D, Byun $H$, et al. White matter change in mesial temporal sclerosis: correlation of MRI with PET, pathology, and clinical features. Epilepsia 1999;40:1634-41

48 Lehericy S, Dormont D, Semah F, et al. Developmental abnormalities of the medial temporal lobe in patients with temporal lobe epilepsy. Am $J$ Neuroradiol 1995; 16:617-26.

49 Meiners L, Van Gils A, Jansen G, et al. Temporal lobe epilepsy: the various appearances of histologically proven mesial temporal sclerosis. Am J Neuroradiol 1994;15:1547-55.

50 Meiners L, Witkamp T, De Kort G, et al. Relevance of temporal lobe white matter changes in hippocampal sclerosis. Magnetic resonance imaging and histology. Invest Radiol 1999;34:38-45.

51 Meiners L, Van Der Grond J, Van Rijen P, et al. Proton magnetic resonance spectroscopy of the temporal lobe white matter in patients with histologically proven hippocampal sclerosis. J Magn Reson Imaging $2000 ; 11: 25-31$

\section{NEUROLOGICAL STAMP}

\section{Eugene Jamot (1879-1937)}

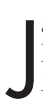

amot received his medical degree from the University of Montpellier. Later, in 1909, he enrolled at the school of tropical medicine in Marseilles. In 1910 he joined the French colonial hygiene group and travelled to Cameroon where Germany had organised a Sleeping Sickness Treatment Research Group. A study he made in 1929 with many colleagues showed a large proportion of the population was affected. Jamot discovered that the tsetse fly harboured trypanosomes and transmitted the disorder. Following measures taken against insect borne infections during the construction of the Panama Canal, Jamot started a successful campaign to wipe out the tsetse fly in Cameroon and succeeded in the prevention of sleeping sickness. Antiparasitic drugs are now available.

Later Jamot became director of the Pasteur Institute at Brazzaville. He returned to France after his retirement and died in Sardent, Creuse, France, where he was born. In 1979, to commemorate the centenary of his birth, Cameroon issued a stamp. This shows both a map of Cameroon and the location of Yadunde and Ayos where he carried out his research, including that of the tsetse fly Glossina palpalis. The stamp also has an inscription "Conqueror of Sleeping Sickness". ( Stanley Gibbons no 865, Scott no 659). The disease remains a major health problem in many African countries. Animal trypanosomiasis is also an important economic and social problem. Vast areas of Africa are unused because of the presence of tsetse flies.

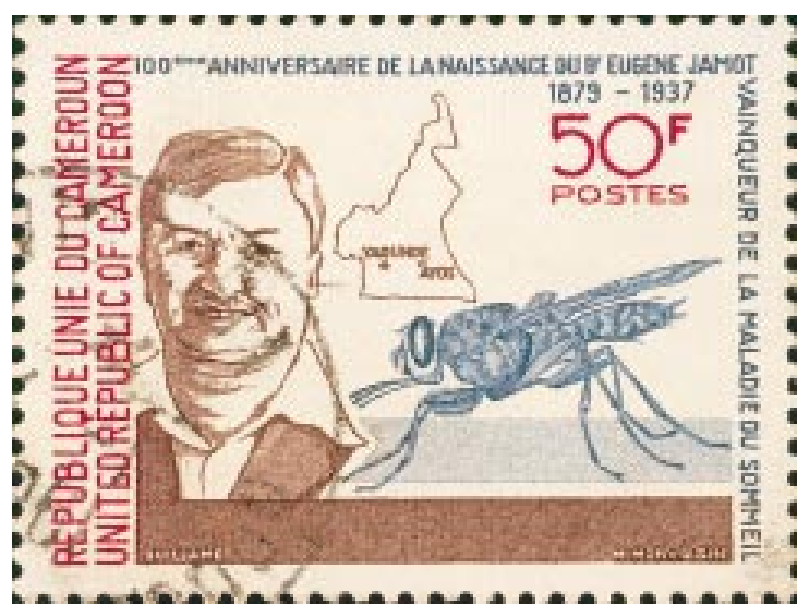

L F Haas 